\title{
ORIGINALS
}

\section{Blood-Glucose, Plasma-Insulin and Growth Hormone Concentrations of Individuals with Minor Abnormalities of Glucose Tolerance}

\author{
C.N. Hales, F.C. Greenwood, F.L. Mitcheld and W.T. Strauss
}

\begin{abstract}
Department of Biochemistry, University of Cambridge; Division of Chemistry and Biochemistry, Imperial Cancer Research Fund, Lincoln's Inn Fields, London W.C. 2;

Department of Clinical Chemistry, University of Edinburgh; Arbroath Infirmary, University of St. Andrews
\end{abstract}

Received July 17, 1967

Summary. 1. The blood-glucose, plasma insulin and growth hormone concentrations have been measured in the fasting state and $30,60,90$ and 120 min after $50 \mathrm{~g}$ glucose administered orally to three groups of subjects: Group I - normal glucose tolerance, no glucosuria; Group II - normal glucose tolerance and glucosuria, Group III - normal fasting blood-sugar concentration but a failure of the blood sugar to return to the fasting level up to $120 \mathrm{~min}$ after glucose. -2 . The influence of weight, sex and age on the results has been analysed. The need for careful matching of subjects with respect to weight, sex and age in as study of changes in blood-glucose, plasma-insulin and growth hormone concentration is emphasized. -3 . The blood-glucose and plasma-insulin concentrations observed in Group II were characterized by an exaggerated early rise and a tendency to late hypoglycaemia. Additional abnormalities were present in this group. - 4. The non-obese subjects of Group III showed a delay in the rise of plasma insulin concentration fol lowing oral glucose and some evidence of resistance to endogenous insulin. The relevance of these findings to the aetiology of diabetes mellitus is discussed.

Concentrations du glucose sanguin, de l'insuline plasmatique et de l'hormone de croissance chez des sujets présentant de légères anomalies de la tolérance au glucose.

Résumé. 1. Les concentrations de glucose sanguin, d'insuline plasmatique et d'hormone de croissance ont été mesurées à l'état de jeûne et $30,60,90$ et $120 \mathrm{~min}$ après administration orale de $50 \mathrm{~g}$ de glucose à trois groupes de sujets: Groupe I: tolérance normale au glucose, pas de glucosurie; groupe II: tolérance normale au glucose et glucosurie; groupe III : glycémie normale à jeun, mais sans retour de la glycémie à sa valeur à jeun, dans les $120 \mathrm{~min}$ qui suivent l'administration de glucose. -2 . L'influence du poids, du sexe et de l'âge sur les résultats a été ana. lysée. On souligne la nécessité de grouper soigneusement les sujets en ce qui concerne le poids, le sexe et l'âge, lors d'une étude sur les variations de concentration du glucose sanguin, de l'insuline plasmatique et de l'hormone de croissance. - 3. Les concentrations de glucose sanguin et d'insuline plasmatique, observées dans le groupe II étai- ent caractérisées par une élévation précoce exagérée et une tendance è une hypoglycémio tardive. D'autres anomalies étaient présentes dans ce groupe. - 4. Les sujets non-obèses du groupe III montraient un retard dans l'élévation de la concentration d'insuline plasmatique, après administration orale de glucose ainsi que des signes évidents de résistance à l'insuline endogène. La relation entre ces faits et l'étiologie du diabète sucré est discutée.

Blutzucker, Plasmainsulin- und WachstumshormonKonzentrationen bei Personen mit leichten Anomalien der Glucose-Toleranz

Zusammenfassung. 1. Die Konzentrationen des Blutzuckers, des Plasmainsulins und des Wachstumshormons wurden nüchtern und $30,60,90$ und $120 \mathrm{~min}$ nach einer oral erfolgten Glucosebelastung mit $50 \mathrm{~g}$ bei 3 Gruppen untersucht: Gruppe I: Normale Glucosetoleranz, keine Glykosurie. Gruppe II : Normale Glucosetoleranz und Glykosurie. Gruppe III: Normaler Nüchternblutzucker; je: doch kehrte der Blutzucker in dieser Gruppe bis zu 120 min nach der Belastung nicht zum Nüchternspiegel zurück. - 2. Der Einfluß des Gewichtes, des Geschlechtes und des Alters wurde analysiert. Die Notwendigkeit, bei diesen Personen Gewicht, Geschlecht und Alter bei einer Untersuchung über die Veränderungen von Blutzucker, Plasmainsulin und Wachstumshormon ins rechte Verhältnis zusetzen, wird betont. - 3. Die Blutzucker- und Plasmainsulinkonzentrationen, die in Gruppe II beobachtet wurden, waren durch einen ausgesprochen frühen Anstieg und eine Tendenz zur Späthypoglykz̈mie gekennzeichnet. Außerdem waren in dieser Gruppe zusätzliche Abnormalitäten festzustellen. - 4. Die nicht.fettleibigen Personen der Gruppe III zeigten einen verzögerten Anstieg der Plasmainsulinkonzentration nach oraler Glucosebelastung. AuBerdem ergab sich offensichtlich eine Resistenz gegenüber endogenem Insulin. Die Bedeutung dieser Befunde wird im Hinblick auf die Pathogenese des Diabetes mellitus diskutiert.

Key-words: Glucose tolerance, glucosuria, blood glucose, plasma insulin, growth hormone, diabetes survey.
The rise of plasma insulin concentration after oral glucose has been found to be delayed in diabetes mellitus (YALOW and BERSON, 1960) and this abnormality has been observed to increase with increasing severity of the diabetes. (HALES and RANDLE, 1963a). The fasting plasma insulin concentration may be raised in this condition (for review see HaLES, 1967a), and an increased basal output of insulin may be an important factor contributing to the relatively poor insulin re- sponse to oral glucose in diabetes. The object of this investigation was to determine whether subjects with minor abnormalities of glucose tolerance (defined below) but who had a normal fasting blood glucose and plasma insulin concentration showed any abnormality of plasma insulin response to oral glucose. Subjects for investigation were collected during a diabetes survey (Mrtohel and Stradoss, 1964). The opportunity was taken of estimating plasma growth hormone concen- 
trations in view of the well known effect of the latter to produce abnormalities of carbohydrate metabolism, and of investigating subjects with glucosuria but normal glucose tolerance. It has been claimed that as many as $63 \%$ of these individuals may become diabetic (ACKERMAN et al., 1958).

\section{Methods}

Tests on People of Arbroath. A diabetic survey of the population of Arbroath was carried out in November 1962 (Mтtchell and Strauss, 1964). As a result a number of individuals were subjected to a full glucose tolerance test. From the results of these tests subjects were selected for a second glucose tolerance test because they fell into one of three groups: the blood glucose concentration remained $10 \mathrm{mg} / 100 \mathrm{ml}$ or more above the fasting concentration throughout the glucose tolerance test (blood samples were taken fasting and at 30, 60, 90 and 120 min after the glucose load).

A second $50 \mathrm{~g}$ oral glucose tolerance test was carried out 12-16 months later. Ambulant subjects were fasted overnight and rested for $30 \mathrm{~min}$ on arrival at the clinic before commencement of the glucose tolerance test. Venous blood samples were taken for the determination of glucose, insulin and growth hormone. Whilst blood-glucose was determined on all the blood samples, some of the subjects tested were not investigated with respect to both plasma insulin and growth hormone concentrations. The subjects were on their usual diet between the tests. The average Scottish diet contains more than $200 \mathrm{~g}$ of carbohydrate per day. The

Table 1. Blood glucose and plasma insulin and growth hormone concentrations in members of the population of Arbroath during $50 \mathrm{~g}$ glucose-tolerance test

\begin{tabular}{|c|c|c|c|c|c|c|c|c|c|}
\hline \multirow[t]{2}{*}{ Group ${ }^{1}$} & \multirow{2}{*}{$\begin{array}{l}\text { No. of } \\
\text { people }\end{array}$} & \multirow{2}{*}{$\begin{array}{l}\text { Age (yr.) } \\
\text { mean } \\
\text { and } \\
\text { range }\end{array}$} & \multirow{2}{*}{$\begin{array}{l}\text { Sex } \\
(\mathrm{M}: \mathrm{F})\end{array}$} & \multirow{2}{*}{$\begin{array}{l}\text { \% ideal } \\
\text { weight } \\
\text { mean and } \\
\text { range }\end{array}$} & \multirow{2}{*}{$\begin{array}{l}\text { Substance } \\
\text { measured }\end{array}$} & \multicolumn{4}{|c|}{ Concentrations and time after glucose administration } \\
\hline & & & & & & $\begin{array}{l}0 \\
\min \end{array}$ & $\begin{array}{l}60 \\
\min \end{array}$ & $\begin{array}{l}90 \\
\min \end{array}$ & $\begin{array}{l}120 \\
\min \end{array}$ \\
\hline \multirow[t]{3}{*}{$\begin{array}{l}\text { I } \\
\text { Normal }\end{array}$} & \multirow[t]{2}{*}{27} & 44 & $20: 7$ & 110 & $\begin{array}{l}\text { Blood glucose } \\
(\mathrm{mg} \text { per } 100 \mathrm{ml})\end{array}$ & $\begin{array}{cc}67 \pm 2 & 99 \pm 3 \\
(50-83) & (57-130)\end{array}$ & $\begin{array}{l}82 \pm 4 \\
(40-135)\end{array}$ & $\begin{array}{l}71 \pm 4 \\
(20 \pm 112)\end{array}$ & $\begin{array}{c}61 \pm 2 \\
(35 \pm 80)\end{array}$ \\
\hline & & \multicolumn{2}{|l|}{$(24-60)$} & $(90-148)$ & $\begin{array}{l}\text { Plasma insulin } \\
\text { (micro units } \\
\text { per m1) }\end{array}$ & $\begin{array}{cc}23 \pm 2 & 66 \pm 6 \\
(11-44) & (23-160)\end{array}$ & $\begin{array}{l}54 \pm 6 \\
(14-120)\end{array}$ & $\begin{array}{l}45 \pm 7 \\
(10-160)\end{array}$ & $\begin{array}{c}32 \pm 3 \\
(13 \pm 72)\end{array}$ \\
\hline & 28 & $\begin{array}{l}42 \\
(20-58)\end{array}$ & $20: 8$ & $\begin{array}{l}110 \\
(90-151)\end{array}$ & $\begin{array}{l}\text { Plasma growth } \\
\text { hormone } \\
\text { (mug per ml) }\end{array}$ & $\begin{array}{l}5.4 \pm 1.3 \quad 5.5 \pm 1.1 \\
(1.0-32)(1.0-22)\end{array}$ & $\begin{array}{r}4.3 \pm 1.4 \\
(1.0-39)\end{array}$ & $\begin{array}{l}3.1 \pm 0.5 \\
(1.0-14)\end{array}$ & $\begin{array}{l}2.6 \pm 0.3 \\
(1.0-5.8)\end{array}$ \\
\hline $\begin{array}{l}\text { II } \\
\text { Normal }\end{array}$ & 25 & 48 & $25: 0$ & 115 & $\begin{array}{l}\text { Blood glucose } \\
(\mathrm{mg} \text { per } 100 \mathrm{ml})\end{array}$ & $\begin{array}{cc}67 \pm 2 & 109 \pm 4 \\
(55-84) & (69-141)\end{array}$ & $\begin{array}{c}101 \pm 5^{3} \\
(58-152)\end{array}$ & $\begin{array}{l}71 \pm 4 \\
(41-105)\end{array}$ & $\begin{array}{l}54 \pm 3 \\
(30-84)\end{array}$ \\
\hline \multirow[t]{2}{*}{$\begin{array}{l}\text { glucos- } \\
\text { uric }\end{array}$} & & \multicolumn{2}{|l|}{$(16-69)$} & $(94-161)$ & $\begin{array}{l}\text { Plasma insulin } \\
\text { (micro units } \\
\text { per ml) }\end{array}$ & $\begin{array}{cc}28 \pm 2 & 88 \pm 9 \\
(15-53) & (18-210)\end{array}$ & $\begin{array}{l}85 \pm 8 \\
(28-162)\end{array}$ & $\begin{array}{l}55+6 \\
(24-184)\end{array}$ & $\begin{array}{c}32 \pm 2 \\
(10-47)\end{array}$ \\
\hline & 18 & $\begin{array}{c}46 \\
(16-69)\end{array}$ & $18: 0$ & $\begin{array}{l}116 \\
(94-161)\end{array}$ & $\begin{array}{l}\text { Plasma growth } \\
\text { hormone } \\
\text { (mug per } \mathrm{ml})\end{array}$ & $\begin{array}{cl}1.9 \pm 0.5^{2} & 2.5 \pm 0.8 \\
(1.0-9.8) & (1.0-13)\end{array}$ & $\begin{array}{l}2.1 \pm 0.6 \\
(1.0-12)\end{array}$ & $\begin{array}{l}2.6 \pm 1.3 \\
(1.0-24)\end{array}$ & $\begin{array}{l}2.6 \pm 0.9 \\
(1.0-15)\end{array}$ \\
\hline \multirow{3}{*}{$\begin{array}{l}\text { III } \\
\text { Delayed } \\
\text { return }\end{array}$} & \multirow[t]{2}{*}{26} & \multirow[t]{2}{*}{$\begin{array}{l}51 \\
(20-70)\end{array}$} & \multirow[t]{2}{*}{$9: 17$} & \multirow[t]{2}{*}{$\begin{array}{l}110 \\
(80-164)\end{array}$} & $\begin{array}{l}\text { Blood glucose } \\
\text { (mg per } 100 \mathrm{ml} \text { ) }\end{array}$ & $\begin{array}{cl}64 \pm 2 & 108 \pm 4 \\
(42 \pm 77) & (65-150)\end{array}$ & $\begin{array}{l}114 \pm 4^{4} \\
(68-155)\end{array}$ & $\begin{array}{l}105 \pm 4^{4} \\
(66-150)\end{array}$ & $\begin{array}{c}91 \pm 3^{4} \\
(62-140)\end{array}$ \\
\hline & & & & & $\begin{array}{l}\text { Plasma insulin } \\
\text { (micro units } \\
\text { per ml) }\end{array}$ & $\begin{array}{cc}23 \pm 2 & 50 \pm 5 \\
(12-60) & (22-97)\end{array}$ & $\begin{array}{l}56 \pm 5 \\
(22 \pm 160)\end{array}$ & $\begin{array}{c}48 \pm 4 \\
(28-100)\end{array}$ & $\begin{array}{c}46 \pm 4^{3} \\
(16-110)\end{array}$ \\
\hline & 26 & $\begin{array}{l}45 \\
(11-70)\end{array}$ & $9: 17$ & $\begin{array}{l}109 \\
(83-164)\end{array}$ & $\begin{array}{l}\text { Plasma growth } \\
\text { hormone } \\
\text { (mug per ml) }\end{array}$ & $\begin{array}{r}9.7 \pm 2.0 \quad 7.3 \pm 1.7 \\
(1.0-35)(1.0-36)\end{array}$ & $\begin{array}{r}5.9 \pm 1.7 \\
(1.0 \pm 31)\end{array}$ & $\begin{array}{r}4.2 \pm 0.8 \\
(1.0-15)\end{array}$ & $\begin{array}{r}3.4 \pm 0.6 \\
(1.0=12)\end{array}$ \\
\hline
\end{tabular}

1 the composition of the groups for which plasma growth hormone was estimated was slightly different (see Text).

a $P<0.05$. $\quad{ }^{3} P<0.01$. $\quad{ }_{1} P \geqq 0.001$ in comparison with the corresponding concentration in Group $\mathrm{I}$.

Group I: (normal) normal glucose tolerance without coincident glucosuria

Group II: (normal glucosuric) normal glucose tolerance with coincident glucosuria

Group III : (delayed return) normal fasting blood glucose concentration; resultis of oral glucose tolerance tests are not very reproducible (MoDonald et al., 1965) and the results of the second glucose tolerance test did not always agree with those of the first. The subjects were therefore regrouped according to the result of the second glucose tolerance test by the criteria above. The results of this test agreed with those of the first in $77 \%, 86 \%$ and $74 \%$ of the subjects previously in Groups I, II 
and III respectively. This paper is concerned solely with the results of the second glucose tolerance test.

The incidence of a positive family history of diabetes was very similar in the three Groups being ${ }^{3} / 27 ;{ }^{2} / 25$ and ${ }^{4} / 26$ in Groups I, II and III respectively (Table 1 ).
(1963) using the 'Auto Analyzer' (Technicon). Insulin was estimated twice by method $\mathrm{C}$ of HALES and RANDLE (1963b). ${ }^{131} I$-Insulin was obtained from The Radiochemical Centre, Amersham, Bucks.

Growth Hormone was estimated by the method of

Table 2. Effect of obesity on blood glucose and plasma insulin concentrations in members of the population of Arbroath during $50 \mathrm{~g}$ glucose-tolerance test

\begin{tabular}{|c|c|c|c|c|c|c|c|c|c|c|}
\hline \multirow[t]{2}{*}{ Group } & \multirow{2}{*}{$\begin{array}{l}\text { No. of } \\
\text { people }\end{array}$} & \multirow{2}{*}{$\begin{array}{l}\text { Age } \\
\text { (yr.) } \\
\text { mean } \\
\text { and } \\
\text { range }\end{array}$} & \multirow{2}{*}{$\begin{array}{l}\text { Sex } \\
(\mathrm{M}: \mathrm{F})\end{array}$} & \multirow{2}{*}{$\begin{array}{l}\% \text { ideal } \\
\text { weight } \\
\text { mean } \\
\text { and range }\end{array}$} & \multirow{2}{*}{$\begin{array}{l}\text { Substance } \\
\text { measured }\end{array}$} & \multicolumn{5}{|c|}{ Concentration and time after glucose administration } \\
\hline & & & & & & $\begin{array}{l}0 \\
\text { min }\end{array}$ & $\begin{array}{l}30 \\
\min \end{array}$ & $\begin{array}{l}60 \\
\min \end{array}$ & $\begin{array}{l}90 \\
\text { min }\end{array}$ & $\begin{array}{l}120 \\
\min \end{array}$ \\
\hline \multirow{2}{*}{$\begin{array}{l}\text { Normal } \\
\text { non } \\
\text { obese }\end{array}$} & 14 & $\begin{array}{l}40 \\
(30-57)\end{array}$ & $10: 4$ & $\begin{array}{l}98 \\
(90-109)\end{array}$ & $\begin{array}{l}\text { Blood glucose } \\
(\mathrm{mg} \text { per } 100 \mathrm{ml})\end{array}$ & $\begin{array}{c}65 \pm 2 \\
(50 \pm 76)\end{array}$ & $\begin{array}{l}91 \pm 4 \\
(57-110)\end{array}$ & $\begin{array}{l}72 \pm 5 \\
(40-102)\end{array}$ & $\begin{array}{c}60 \pm 5 \\
(20-90)\end{array}$ & $\begin{array}{c}58 \pm 3 \\
(35-80)\end{array}$ \\
\hline & & & & & $\begin{array}{l}\text { Plasma insulin } \\
\text { (micro units } \\
\text { per ml) }\end{array}$ & $\begin{array}{c}20 \pm 2 \\
(11-31)\end{array}$ & $\begin{array}{r}55 \pm \\
(23\end{array}$ & $\begin{array}{l}44 \pm 7 \\
(14-120)\end{array}$ & $\begin{array}{l}33 \pm 9 \\
(10 \div-140)\end{array}$ & $\begin{array}{c}25 \pm 3 \\
(13-48)\end{array}$ \\
\hline \multirow[t]{2}{*}{$\begin{array}{l}\text { Normal } \\
\text { obese }\end{array}$} & 13 & $\begin{array}{l}48 \\
(24-60)\end{array}$ & $10: 3$ & $\begin{array}{l}123 \\
(110-148)\end{array}$ & $\begin{array}{l}\text { Blood glucose } \\
(\mathrm{mg} \text { per } 100 \mathrm{ml})\end{array}$ & $\begin{array}{c}68 \pm 4 \\
(55-83)\end{array}$ & $\begin{array}{l}106 \pm 4 \\
(85-130)\end{array}$ & $\begin{array}{r}92 \\
(58-\end{array}$ & $\begin{array}{l}83 \pm 4 \\
(65-112)\end{array}$ & $\begin{array}{c}63 \pm 2 \\
(50-76)\end{array}$ \\
\hline & & & & & $\begin{array}{l}\text { Plasma insulin } \\
\text { (micro units } \\
\text { per ml) }\end{array}$ & $\begin{array}{c}27 \pm 2 \\
(18-44)\end{array}$ & $\begin{array}{l}74 \pm \\
(29-\end{array}$ & $\begin{array}{l}65 \pm 8 \\
(23-120)\end{array}$ & $\begin{array}{l}58 \pm 9 \\
(26-160)\end{array}$ & $\begin{array}{c}38 \pm 4 \\
(17-72)\end{array}$ \\
\hline \multirow{2}{*}{$\begin{array}{l}\text { Normal } \\
\text { glucos- } \\
\text { uric } \\
\text { non } \\
\text { obese }\end{array}$} & 10 & $\begin{array}{l}48 \\
(16-67)\end{array}$ & $10: 0$ & $\frac{101}{(94-108)}$ & $\begin{array}{l}\text { Blood glucose } \\
\text { (mg per } 100 \mathrm{ml})\end{array}$ & $\begin{array}{c}66 \pm 2 \\
(55-74)\end{array}$ & $\begin{array}{l}108 \pm 6^{1} \\
(81-132)\end{array}$ & $\begin{array}{l}84 \pm 6 \\
(58-112)\end{array}$ & $\begin{array}{c}60 \pm 5 \\
(41-93)\end{array}$ & $\begin{array}{l}49 \pm 4 \\
(36-79)\end{array}$ \\
\hline & & & & & $\begin{array}{l}\text { Plasma insulin } \\
\text { (micro units } \\
\text { per ml) }\end{array}$ & $\begin{array}{c}27 \pm 3^{1} \\
(15-41)\end{array}$ & $\begin{array}{l}88 \pm 19 \\
(18-210)\end{array}$ & $\begin{array}{c}72 \pm 12^{1} \\
(28-150)\end{array}$ & $\begin{array}{c}42 \pm 4 \\
(24-61)\end{array}$ & $\begin{array}{l}31 \pm 2 \\
(19-47)\end{array}$ \\
\hline \multirow{2}{*}{$\begin{array}{l}\text { Normal } \\
\text { glucos- } \\
\text { uric } \\
\text { obese }\end{array}$} & 14 & $\begin{array}{l}47 \\
(25-69)\end{array}$ & $14: 0$ & $\begin{array}{l}127 \\
(110-161)\end{array}$ & $\begin{array}{l}\text { Blood glucose } \\
(\mathrm{mg} \text { per } 100 \mathrm{ml})\end{array}$ & $\begin{array}{c}67 \pm 3 \\
(55 \pm 84)\end{array}$ & $\begin{array}{l}108 \pm 5 \\
(69-141)\end{array}$ & $\begin{array}{l}112 \pm 6^{1} \\
(75-152)\end{array}$ & $\begin{array}{l}78 \pm 5 \\
(42-105)\end{array}$ & $\begin{array}{c}58 \pm 4 \\
(30-84)\end{array}$ \\
\hline & & & & & $\begin{array}{l}\text { Plasma insulin } \\
\text { (micro units } \\
\text { per ml) }\end{array}$ & $\begin{array}{c}28 \pm 3 \\
(18-53)\end{array}$ & $\begin{array}{l}89 \pm \\
(46-\end{array}$ & $\begin{array}{c}94 \pm 6^{1} \\
(40-162)\end{array}$ & $\begin{array}{c}64 \pm 10 \\
(26-184)\end{array}$ & $\begin{array}{l}32 \pm 3 \\
(10-47)\end{array}$ \\
\hline \multirow{2}{*}{$\begin{array}{l}\text { Delayed } \\
\text { return } \\
\text { non } \\
\text { obese }\end{array}$} & 14 & $\begin{array}{l}46 \\
(20-67)\end{array}$ & $5: 9$ & $\begin{array}{l}95 \\
(80-109)\end{array}$ & $\begin{array}{l}\text { Blood glucose } \\
\text { (mg per } 100 \mathrm{ml})\end{array}$ & $\begin{array}{c}66 \pm 2 \\
(48-77) \\
\end{array}$ & $\begin{array}{l}104 \pm 5 \\
(65-130)\end{array}$ & $\begin{array}{l}117 \pm 6 \\
(74-155) \\
\end{array}$ & $\begin{array}{l}107 \pm 6 \\
(74-150)\end{array}$ & $\begin{array}{l}(93 \pm 5 \\
(68-140)\end{array}$ \\
\hline & & & & & $\begin{array}{l}\text { Plasma insulin } \\
\text { (micro units } \\
\text { per ml) }\end{array}$ & $\begin{array}{c}18 \pm 2 \\
(12-35)\end{array}$ & $\begin{array}{c}42 \pm 41 \\
(22-64)\end{array}$ & $\begin{array}{c}46 \pm 4 \\
(22 \pm-75)\end{array}$ & $\begin{array}{c}48 \pm 5 \\
(28-93)\end{array}$ & $\begin{array}{l}42 \pm 4^{2} \\
(16-75)\end{array}$ \\
\hline \multirow[t]{2}{*}{$\begin{array}{l}\text { Delayed } \\
\text { return } \\
\text { obese }\end{array}$} & 12 & $\begin{array}{l}57 \\
(34-70)\end{array}$ & $4: 8$ & $\begin{array}{l}129 \\
(110-164)\end{array}$ & $\begin{array}{l}\text { Blood glucose } \\
(\mathrm{mg} \text { per } 100 \mathrm{ml})\end{array}$ & $\begin{array}{c}62 \pm 3 \\
(42-74) \\
\end{array}$ & $\begin{array}{l}112 \pm 6 \\
(72-150)\end{array}$ & $\begin{array}{l}111 \pm 6 \\
(68-140)\end{array}$ & $\begin{array}{l}103 \pm 5 \\
(66-127)\end{array}$ & $\begin{array}{l}89 \pm 4 \\
(62 \pm 106)\end{array}$ \\
\hline & & & & & $\begin{array}{l}\text { Plasma insulin } \\
\text { (micro units) }\end{array}$ & $\begin{array}{l}28 \pm 3 \\
(17-60)\end{array}$ & $\begin{array}{c}61 \pm 6 \\
(29-97)\end{array}$ & $\begin{array}{c}61 \pm 10 \\
(33-160)\end{array}$ & $\begin{array}{l}57 \pm 6 \\
(32-100)\end{array}$ & $\begin{array}{c}56 \pm 8^{1} \\
(20-110)\end{array}$ \\
\hline
\end{tabular}

$1 P<0.05$.

$2 P<0.01$.

${ }^{3} P<0.001$ in comparison with the corresponding concentration in Group I.

Plasma samples. Blood was drawn from an antecubital vein while fasting and 30, 60,90 and 120 min after the administration of glucose and delivered into tubes containing heparin. Plasma was separated at once by centrifugation and for the determination of insulin and growth hormone concentration, was stored at $-20^{\circ} \mathrm{C}$ prior to assay.

Blood or Plasma Estimations. Blood glucose was estimated by the glucose-oxidase method of DIscombe
HUNTER and Greenwood (1964) using a laboratory standard (ICRF Batch 6 Peak 2) immunologically $150 \%$ of the MRC Standard A preparation of human growth hormone.

\section{Results}

The blood-glucose, plasma insulin and growth hormone concentrations (mean \pm standard error and range) during the second glucose tolerance test together 
with the size and age, sex and weight compositions of the groups are given in Table 1. The figures for ideal weight in relation to age height and sex were taken from Kemst.ey (1951-52). The blood-glucose concentrations refer to the subjects for whom the plasma insulin was estimated. The blood glucose concentration of the subjects for whom plasma growth hormone was estimated did not differ significantly from the former and have not therefore been quoted.

In order to determine the effects of weight, sex and age on the parameters studied the groups were subdivided. Due to the different compositions of the groups the blood glucose and plasma insulin concentrations were considered separately from the plasma growth hormone.

Weight - each group was divided into a non-obese $(\leq 109 \%$ ideal weight) and an obese $(\geq 110 \%$ ideal weight) subgroup. The blood-glucose and plasma insulin concentrations (mean \pm standard error and range) together with the compositions of the subgroups are given in Table 2. The plasma growth hormone concentrations are given in Table 3 .

Sex - Group I and Group III were subdivided into female and male subgroups, the latter being matched so that the mean weight and age of the subgroups was approximately the same, in order to determine whether the plasma glucose and insulin results varied according to the sex of the subjects. The blood-glucose and plasma insulin concentrations (mean \pm standard error and range) together with the compositions of the subgroups are given in Table 4. The plasma growth hormone concentrations were subdivided according to both weight and sex and the results are given in Table 3 .

Age - the individuals for whom blood-glucose and plasma insulin concentration were determined were subdivided into "young" (40 years and under) and "old" (51 years and over) subgroups. These subgroups were matched so that the sex and weight compositions of the two subgroups derived from any given group were approximately the same. The mean concentrations ( + standard error and range) together with the compositions of the subgroups are given in Table 5 .

Blood-glucose - The mean blood-glucose concentration during the glucose tolerance test (Table 1) was higher at 30 and $60 \mathrm{~min}$ and lower at $120 \mathrm{~min}$ after glucose in Group II than in Group I, but only the difference at $60 \mathrm{~min}$ was statistically significant $(P<0.01)$. The mean blood-glucose concentration was higher in Group III than in Group I at 30, 60, 90 and 120 min after glucose, the latter three differences being significant $(P<0.001)$.

Weight - the mean blood-glucose concentration was significantly higher (Table 2): a) in the obese than in the non obese subgroup I $30(P<0.05) 60(P<0.05)$ and $90(P<0.001)$ min after glucose; $b)$ in the obese than in the non obese subgroup of Group II 60 $(P<0.01)$ and $90(P<0.05)$ min after glucose. The differences observed when the pooled results of Group I were compared with those of Group II were also ob- served when their respective non-obese and obese subgroups were compared (Table 2 ), but were significant at $30 \mathrm{~min}(P<0.05)$ in the non-obese and at $60 \mathrm{~min}$ $(P<0.05)$ in the obese subgroup comparisons. The differences observed when the pooled results of Group I were compared with those of Group III were also observed when their respective non-obese and obese subgroups were compared (Table 2). The mean blood glucose and plasma insulin concentrations of the non-obese subgroups of Groups I, II and III are plotted in Fig. 1.

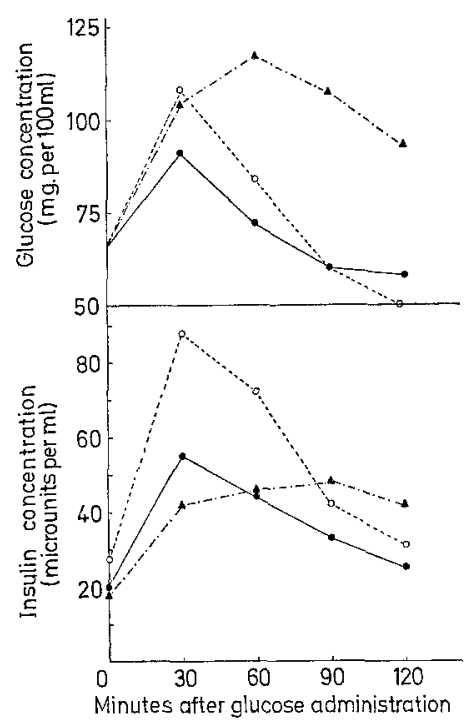

Fig. 1. Mean blood glucose and plasma insulin concentrations during $50 \mathrm{~g}$ oral glucose-tolerance tests in nonobese normal people (Group I), people with glycosuria (Group II) and people with a minor abnormality of glucose tolerance (Group III)

Abscissa: lower graph; Minutes after glucose administration; $0,30,60,90,120$.

Ordinate: upper graph; Glucose concentration (mg per $100 \mathrm{ml}) 50,75,100,125$.

lower graph; Insulin concentration (microunits per ml) $0,20,40,6080$.

normal people (Group I)

$\ldots$ people with glycosuria (Group II)

_._... people with a minor abnormality of glucose tolerance (Group III)

Sex - no significant differences were observed when the female was compared with the male subgroup of Group I or Group III (Table 4). Group II contained only males.

Age - the mean blood-glucose concentration was significantly higher (Table 5) in the old than in the young subgroup of Group I $90 \mathrm{~min}$ after glucose $(P<0.05)$, and in the old than in the young subgroup of Group III $60(P<0.05), 90(P<0.01)$ and 120 $(P<0.01)$ min after glucose.

Plasma insulin. The mean plasma-insulin was higher (Table 1) in: a) Group II than in Group I, 30 and $60 \mathrm{~min}$ after glucose, the latter difference being statistically significant $(P<0.01)$; b) Group III than in Group I $120 \mathrm{~min}$ after glucose $(P<0.01)$. 
Table 3. Plasma growth hormone concentrations* in members of the population of Arbroath during $50 \mathrm{~g}$ glucose-tolerance test

\begin{tabular}{|c|c|c|c|c|c|c|c|c|c|}
\hline Group & Sex & $\begin{array}{l}\text { No. of } \\
\text { people }\end{array}$ & $\begin{array}{l}\text { Age (yr.) } \\
\text { mean and } \\
\text { range }\end{array}$ & $\begin{array}{l}\% \text { ideal } \\
\text { weight mean } \\
\text { and range }\end{array}$ & $\begin{array}{l}\text { Plasma } \\
0 \\
\text { min }\end{array}$ & $\begin{array}{l}\text { centration as } \\
30 \\
\text { min }\end{array}$ & $\begin{array}{l}\text { d time aft } \\
60 \\
\text { min. }\end{array}$ & $\begin{array}{l}\text { glucose a } \\
90 \\
\text { min }\end{array}$ & $\begin{array}{l}\text { ainistration } \\
120 \\
\min \end{array}$ \\
\hline \multirow[t]{2}{*}{$\begin{array}{l}\text { Normal } \\
\text { non-obese }\end{array}$} & female & 5 & $\begin{array}{l}44 \\
(33-53)\end{array}$ & $\begin{array}{r}93 \\
(86-97)\end{array}$ & $\begin{array}{c}16 \pm 5 \\
(2.9-32)\end{array}$ & $\begin{array}{c}12 \pm 3 \\
(4.0-22)\end{array}$ & $\begin{array}{l}3.6 \pm 0.7 \\
(2.1-5.9)\end{array}$ & $\begin{array}{c}3.2 \pm 0.6 \\
(1.0-4.1)\end{array}$ & $\begin{array}{c}3.4 \pm 0.8 \\
(1.0-5.8)\end{array}$ \\
\hline & male & 11 & $\begin{array}{l}37 \\
(20-55)\end{array}$ & $\begin{array}{l}101 \\
(90-109)\end{array}$ & $\begin{array}{c}2.4 \pm 0.5 \\
(1.0 \pm 5.6)\end{array}$ & $\begin{array}{c}2.2 \pm 0.3 \\
(1.0-4.2)\end{array}$ & $\begin{array}{c}2.0 \pm 0.3 \\
(1.0-3.7)\end{array}$ & $\begin{array}{c}2.1 \pm 0.3 \\
(1.0 \pm 3.6)\end{array}$ & $\begin{array}{c}2.0 \pm 0.3 \\
(1.0-4.4)\end{array}$ \\
\hline \multirow[t]{2}{*}{$\begin{array}{l}\text { Normal } \\
\text { obese }\end{array}$} & female & 3 & $\begin{array}{l}47 \\
(33-58)\end{array}$ & $\begin{array}{l}125 \\
(116-140)\end{array}$ & $\begin{array}{l}6.7 \\
(6.1-7.1)\end{array}$ & $\begin{array}{l}14 \\
(9.5-18)\end{array}$ & $\begin{array}{l}19 \\
(2.9-39)\end{array}$ & $\begin{array}{l}6.4 \\
(2.8-14)\end{array}$ & $\begin{array}{l}2.9 \\
(1.9-4.0)\end{array}$ \\
\hline & male & 9 & $\begin{array}{l}45 \\
(24-54)\end{array}$ & $\begin{array}{l}126 \\
(110-151)\end{array}$ & $\begin{array}{c}2.7 \pm 0.6 \\
(1.0-6.8)\end{array}$ & $\begin{array}{c}2.9 \pm 0.7 \\
(1.0-7.8)\end{array}$ & $\begin{array}{c}2.5 \pm 0.6 \\
(1.0 \pm 5.3)\end{array}$ & $\begin{array}{c}3.1 \pm 0.9 \\
(1.0-8.8)\end{array}$ & $\begin{array}{c}2.6 \pm 0.6 \\
(1.0-5.4)\end{array}$ \\
\hline \multirow{2}{*}{$\begin{array}{l}\text { Normal } \\
\text { glucosuric } \\
\text { non-obese } \\
\text { Normal } \\
\text { glucosuric } \\
\text { obese }\end{array}$} & male & 6 & $\begin{array}{l}47 \\
(16-67)\end{array}$ & $\begin{array}{l}101 \\
(94-105)\end{array}$ & $\begin{array}{c}2.8 \pm 1.4 \\
(1.0-9.8)\end{array}$ & $\begin{array}{c}4.8 \pm 2.3 \\
(1.0-13.3)\end{array}$ & $\begin{array}{c}3.3 \pm 1.7 \\
(1.0-12)\end{array}$ & $\begin{array}{c}4.9 \pm 3.9 \\
(1.0-24)\end{array}$ & $\begin{array}{r}3.4 \pm 2.3 \\
(1.0-15)\end{array}$ \\
\hline & male & 12 & $\begin{array}{l}46 \\
(25-69)\end{array}$ & $\begin{array}{l}125 \\
(110-161)\end{array}$ & $\begin{array}{c}1.6 \pm 0.1^{1} \\
(1.0-2.5)\end{array}$ & $\begin{array}{c}1.5 \pm 0.1^{1} \\
(1.0-2.5)\end{array}$ & $\begin{array}{c}1.6 \pm 0.1 \\
(1.0-2.4)\end{array}$ & $\begin{array}{c}1.4 \pm 0.1^{1} \\
(1.0-2.3)\end{array}$ & $\begin{array}{c}2.0 \pm 0.7 \\
(1.0-9.0)\end{array}$ \\
\hline \multirow[t]{2}{*}{$\begin{array}{l}\text { Delayed } \\
\text { return } \\
\text { non-obese }\end{array}$} & female & 11 & $\begin{array}{l}40 \\
(11-64)\end{array}$ & $\begin{array}{r}96 \\
(83-109) \\
\end{array}$ & $\begin{array}{r}12 \pm 3.3 \\
(1.4-35) \\
\end{array}$ & $\begin{array}{r}6.4 \pm 1.5 \\
(1.0-14) \\
\end{array}$ & $\begin{array}{c}3.1 \pm 0.5 \\
(1.1-5.4)\end{array}$ & $\begin{array}{c}2.5 \pm 0.2 \\
(1.0-3.2) \\
\end{array}$ & $\begin{array}{c}2.4 \pm 0.2 \\
(1.0-3.4) \\
\end{array}$ \\
\hline & male & 5 & $\begin{array}{l}47 \\
(36-65)\end{array}$ & $\begin{array}{l}97 \\
(80-109)\end{array}$ & $\begin{array}{c}17 \pm 5.8^{2} \\
(1.6-29)\end{array}$ & $\begin{array}{c}13 \pm 7.2^{1} \\
(1.7-36)\end{array}$ & $\begin{array}{c}12 \pm 5.3^{1} \\
(1.9-31)\end{array}$ & $\begin{array}{c}7.5 \pm 2.2^{2} \\
(2.0-15)\end{array}$ & $\begin{array}{r}6.3 \pm 1.3^{2} \\
(1.7-9.5)\end{array}$ \\
\hline \multirow{2}{*}{$\begin{array}{l}\text { Delayed } \\
\text { return } \\
\text { obese }\end{array}$} & fernale & 6 & $\begin{array}{l}48 \\
(15-62)\end{array}$ & $\begin{array}{l}135 \\
(116-164)\end{array}$ & $\begin{array}{c}4.3 \pm 1.2 \\
(1.8-9.9)\end{array}$ & $\begin{array}{c}3.7 \pm 1.0 \\
(1.4-8.6)\end{array}$ & $\begin{array}{c}2.0 \pm 0.5 \\
(1.0 \pm 3.9)\end{array}$ & $\begin{array}{c}2.0 \pm 0.7 \\
(1.0-4.9)\end{array}$ & $\begin{array}{c}2.1 \pm 0.6 \\
(1.0=4.3)\end{array}$ \\
\hline & male & 4 & $\begin{array}{l}53 \\
(33-70)\end{array}$ & $\begin{array}{l}116 \\
(110-119)\end{array}$ & $\begin{array}{c}2.3 \pm 0.7 \\
(1.0-3.6)\end{array}$ & $\begin{array}{c}2.3 \pm 0.1 \\
(2.0-2.8)\end{array}$ & $\begin{array}{r}11 \pm 8.4 \\
(2.3-28)\end{array}$ & $\begin{array}{r}7.3 \pm 3.3 \\
(1.0-13)\end{array}$ & $\begin{array}{l}4.3 \pm 2.6 \\
(1.0-12.1)\end{array}$ \\
\hline
\end{tabular}

I $P<0.05$.

${ }^{2} P<0.01$ in comparison with the corresponding concentration in Group $I$.

$*(\mathrm{~m} \mu \mathrm{g} / \mathrm{ml})$

Table 4. Blood glucose and plasma insulin concentrations during $50 \mathrm{~g}$ glucose tolerance tesis on male and female members of the population of Arbroath

\begin{tabular}{|c|c|c|c|c|c|c|c|c|c|}
\hline \multirow[t]{2}{*}{ Group } & \multirow{2}{*}{$\begin{array}{l}\text { No. of } \\
\text { people }\end{array}$} & \multirow{2}{*}{$\begin{array}{l}\text { Age (yr.) } \\
\text { mean and } \\
\text { range }\end{array}$} & \multirow{2}{*}{$\begin{array}{l}\% \text { ideal } \\
\text { weight } \\
\text { mean and } \\
\text { range }\end{array}$} & \multirow{2}{*}{$\begin{array}{l}\text { Substance } \\
\text { measured }\end{array}$} & \multicolumn{5}{|c|}{ Concentration and time after glucose administration } \\
\hline & & & & & $\begin{array}{l}0 \\
\min \end{array}$ & $\begin{array}{l}30 \\
\min \end{array}$ & $\begin{array}{l}60 \\
\min \end{array}$ & $\begin{array}{l}90 \\
\min \end{array}$ & $\begin{array}{l}120 \\
\min \end{array}$ \\
\hline \multirow[t]{2}{*}{$\begin{array}{l}\text { Normal } \\
\text { female }\end{array}$} & 7 & $\begin{array}{c}45 \\
(33-58)\end{array}$ & $\begin{array}{l}107 \\
(91-140)\end{array}$ & $\begin{array}{l}\text { Blood glucose } \\
(\mathrm{mg} \text { per } 100 \mathrm{ml})\end{array}$ & $\begin{array}{c}69 \pm 3 \\
(55 \pm 76)\end{array}$ & $\begin{array}{l}103 \pm 8 \\
(65-130)\end{array}$ & $\begin{array}{l}79 \pm 7 \\
(55-103)\end{array}$ & $\begin{array}{c}71 \pm 12 \\
(53 \pm 90)\end{array}$ & $\begin{array}{c}63 \pm 9 \\
(56-74)\end{array}$ \\
\hline & & & & $\mathrm{ml}$ ) & $\begin{array}{l}23+ \\
(19\end{array}$ & $\begin{array}{r}59 \\
(44-\end{array}$ & $\begin{array}{r}50 \\
(39\end{array}$ & $\begin{array}{l}32 \pm \\
(12 \pm\end{array}$ & $\begin{array}{c}35 \pm 8 \\
(17-72)\end{array}$ \\
\hline \multirow[t]{2}{*}{$\begin{array}{l}\text { Normal } \\
\text { male }\end{array}$} & 20 & $\begin{array}{l}44 \\
(24-60)\end{array}$ & $\begin{array}{l}111 \\
(90-148)\end{array}$ & $\begin{array}{l}\text { Blood glucose } \\
(\mathrm{mg} \text { per } 100 \mathrm{mI})\end{array}$ & $\begin{array}{c}65 \pm 2 \\
(50-83)\end{array}$ & $\begin{array}{c}96 \pm 3 \\
(57 \pm 120)\end{array}$ & $\begin{array}{l}82 \pm 6 \\
(40 \pm 135)\end{array}$ & $\begin{array}{l}71 \pm 5 \\
(20-112)\end{array}$ & $\begin{array}{c}59 \pm 3 \\
(35-80)\end{array}$ \\
\hline & & & & $\begin{array}{l}\text { Plasma insulin } \\
\text { (microunits per ml) }\end{array}$ & $\begin{array}{l}23 \pm 2 \\
(11-44)\end{array}$ & $\begin{array}{c}68 \pm 8 \\
(23 \pm 160)\end{array}$ & $\begin{array}{c}55 \pm 8 \\
(14-120)\end{array}$ & $\begin{array}{c}50 \pm 9 \\
(10 \div-160)\end{array}$ & $\begin{array}{c}30 \pm 3 \\
(13-53)\end{array}$ \\
\hline \multirow[t]{2}{*}{$\begin{array}{l}\text { Delayed } \\
\text { return } \\
\text { female }\end{array}$} & 11 & $\begin{array}{l}48 \\
(20-67)\end{array}$ & $\begin{array}{l}103 \\
(88-121)\end{array}$ & $\begin{array}{l}\text { Bloo } \\
\text { (mg } 1\end{array}$ & $\begin{array}{c}61 \pm 4 \\
(42-77) \\
\end{array}$ & $\begin{array}{l}109 \pm 5 \\
(79-130)\end{array}$ & $\begin{array}{l}116 \pm 7 \\
(68-155)\end{array}$ & $\begin{array}{l}113 \pm 8 \\
(66-150)\end{array}$ & $\begin{array}{c}95 \pm 7 \\
(62-140) \\
\end{array}$ \\
\hline & & & & $\begin{array}{l}\text { Plasma insulin } \\
\text { (microunits per ml) }\end{array}$ & $\begin{array}{c}18 \pm 1 \\
(12-28)\end{array}$ & $\begin{array}{r}42 \pm \\
(22\end{array}$ & $\begin{array}{c}52 \pm 4 \\
(38-83)\end{array}$ & $\begin{array}{c}57 \pm 6 \\
(35-100)\end{array}$ & $\begin{array}{l}44 \pm 5 \\
(25-84)\end{array}$ \\
\hline \multirow[t]{2}{*}{$\begin{array}{l}\text { Delayed } \\
\text { return } \\
\text { male }\end{array}$} & 8 & $\begin{array}{c}56 \\
(38-70)\end{array}$ & $\begin{array}{l}103 \\
(83-120)\end{array}$ & $\begin{array}{l}\text { Blood glucose } \\
(\mathrm{mg} \text { per } 100 \mathrm{ml})\end{array}$ & $\begin{array}{c}64 \pm 3 \\
(54-75) \\
\end{array}$ & $\begin{array}{l}108 \pm 9 \\
(65-150)\end{array}$ & $\begin{array}{l}114 \pm 8 \\
(74-136)\end{array}$ & $\begin{array}{l}97 \pm 7 \\
(74-127)\end{array}$ & $\begin{array}{c}86 \pm 3 \\
(68-95)\end{array}$ \\
\hline & & & & $\begin{array}{l}\text { Plasma insulin } \\
\text { (microunits per ml) }\end{array}$ & $\begin{array}{c}23 \pm 2 \\
(15 \div 36)\end{array}$ & $\begin{array}{c}50 \pm 7 \\
(25 \pm 79)\end{array}$ & $\begin{array}{c}49 \pm 7 \\
(22 \pm 75)\end{array}$ & $\begin{array}{c}47 \pm 7 \\
(28-93)\end{array}$ & $\begin{array}{c}45 \pm 7 \\
(16-75)\end{array}$ \\
\hline
\end{tabular}


Weight - the mean plasma-insulin concentration was higher in all the obese subgroups at all stages in the glucose tolerance test (Table 2 ), but significantly so only in: a) Group I fasting and $120 \mathrm{~min}$ after glucose $(P<0.05)$; b) Group III fasting $(P<0.05)$ and $30 \mathrm{~min}$ $(P<0.05)$ after glucose. The differences observed when the pooled results of Group I were compared with those of Group II were also observed when their respective dition, the mean plasma-insulin concentration of the non-obese subgroup of Group III was significantly lower than that of the non obese subgroup of Group I $30 \mathrm{~min}$ after glucose $(P<0.05)$.

Sex - no significant differences were observed.

Age - the mean plasma-insulin concentration was significantly higher in the old subgroup of Group I 90 $(P<0.05)$ and $120(P<0.05)$ min after glucose (Table 5

Table 5. Effect of age on blood glucose and plasma insulin concentration in members of the population of Arbroath during $50 \mathrm{~g}$ glucose-tolerance test

\begin{tabular}{|c|c|c|c|c|c|c|c|c|c|c|}
\hline \multirow[t]{2}{*}{ Group } & \multirow{2}{*}{$\begin{array}{l}\text { No. of } \\
\text { people }\end{array}$} & \multirow{2}{*}{$\begin{array}{l}\text { Age (yr.) } \\
\text { mean } \\
\text { and } \\
\text { range }\end{array}$} & \multirow{2}{*}{$\begin{array}{l}\text { Sex } \\
(M: F)\end{array}$} & \multirow{2}{*}{$\begin{array}{l}\% \text { ideal } \\
\text { weight mean } \\
\text { and range }\end{array}$} & \multirow{2}{*}{$\begin{array}{l}\text { Substance } \\
\text { measured }\end{array}$} & \multicolumn{5}{|c|}{ Concentration and time after glucose administration } \\
\hline & & & & & & $\begin{array}{l}0 \\
\min \end{array}$ & $\begin{array}{l}30 \\
\min \end{array}$ & $\begin{array}{l}60 \\
\min \end{array}$ & $\begin{array}{l}90 \\
\min \end{array}$ & $\begin{array}{l}120 \\
\min \end{array}$ \\
\hline Normal & 12 & $\begin{array}{l}33 \\
(24-40)\end{array}$ & $9: 3$ & $\begin{array}{l}110 \\
(90-148)\end{array}$ & $\begin{array}{l}\text { Blood glucose } \\
(\mathrm{mg} \text { per } 100 \mathrm{ml})\end{array}$ & $\begin{array}{c}69 \pm 2 \\
(54-76)\end{array}$ & $\begin{array}{l}103 \pm 3 \\
(85-120)\end{array}$ & $\begin{array}{l}91 \pm 6 \\
(40-102)\end{array}$ & $\begin{array}{c}64 \pm 6 \\
(20-90)\end{array}$ & $\begin{array}{c}62 \pm 4 \\
(43-80)\end{array}$ \\
\hline young & & & & & $\begin{array}{l}\text { Plasma insulin } \\
\text { (micro units } \\
\text { per ml) }\end{array}$ & $\begin{array}{c}22 \pm 2 \\
(15-33)\end{array}$ & $\begin{array}{r}69 \pm \\
(44-\end{array}$ & $\begin{array}{r}46= \\
(14=\end{array}$ & $\begin{array}{c}31 \pm 5 \\
(12-81)\end{array}$ & $\begin{array}{r}26 \pm \\
(15\end{array}$ \\
\hline$\overline{\text { Norma1 }}$ & 12 & $\begin{array}{l}54 \\
(51-60)\end{array}$ & $10: 2$ & $\begin{array}{l}111 \\
(93-126)\end{array}$ & $\begin{array}{l}\text { Blood glucose } \\
\text { (mg per } 100 \mathrm{ml} \text { ) }\end{array}$ & $\begin{array}{c}65 \pm 3 \\
(50-83)\end{array}$ & $\begin{array}{c}99 \pm 6 \\
(57-130)\end{array}$ & $\begin{array}{l}94 \pm 6 \\
(58-135)\end{array}$ & $\begin{array}{c}80 \pm 5^{1} \\
(52 \pm 112)\end{array}$ & $\begin{array}{c}59 \pm 3 \\
(35-70)\end{array}$ \\
\hline old & & & & & $\begin{array}{l}\text { Plasma insulin } \\
\text { (micro units } \\
\text { per ml) }\end{array}$ & $\begin{array}{l}25 \pm 3 \\
(12-44)\end{array}$ & $\begin{array}{c}68 \pm 12 \\
(25-160)\end{array}$ & $\begin{array}{c}67 \pm 11 \\
(23-120)\end{array}$ & $\begin{array}{c}64 \pm 13^{1} \\
(24-160)\end{array}$ & $\begin{array}{c}40 \pm 4^{1} \\
(27 \pm 72)\end{array}$ \\
\hline $\begin{array}{l}\text { Normal } \\
\text { glucos- } \\
\text { uric }\end{array}$ & 7 & $\begin{array}{l}31 \\
(16-37)\end{array}$ & $7: 0$ & $\begin{array}{l}123 \\
(94-161)\end{array}$ & $\begin{array}{l}\text { Blood } \\
\text { (mg pe }\end{array}$ & $\begin{array}{l}71 \pm 8 \\
(59 \pm 80)\end{array}$ & $\begin{array}{l}110 \pm 10 \\
(82-141)\end{array}$ & $\begin{array}{l}103= \\
(64-\end{array}$ & $\begin{array}{c}71 \pm 8 \\
(42 \pm 103)\end{array}$ & $\begin{array}{c}57 \pm 5 \\
(30 \pm 78)\end{array}$ \\
\hline young & & & & & $\begin{array}{l}\text { Plasma insulin } \\
\text { (micro units } \\
\text { per ml) }\end{array}$ & $\begin{array}{l}34 \pm 6 \\
(22-53)\end{array}$ & $\begin{array}{l}107 \pm 22 \\
(46-200)\end{array}$ & $\begin{array}{l}114 \pm \\
(45-\end{array}$ & $\begin{array}{r}73 \pm \\
(41 \pm\end{array}$ & $\begin{array}{l}34 \pm 5 \\
(10-47)\end{array}$ \\
\hline $\begin{array}{l}\text { Normal } \\
\text { glucos- } \\
\text { uric }\end{array}$ & 13 & $\begin{array}{l}58 \\
(51-69)\end{array}$ & $13: 0$ & $\begin{array}{l}114 \\
(98-133)\end{array}$ & $\begin{array}{l}\text { Blood glucose } \\
\text { (mg per } 100 \mathrm{ml})\end{array}$ & $\begin{array}{c}67 \pm 3 \\
(55-84)\end{array}$ & $\begin{array}{l}106 \pm 6 \\
(69-140)\end{array}$ & $\begin{array}{l}104 \pm 7 \\
(68-150)\end{array}$ & $\begin{array}{l}73 \pm 7 \\
(41-105)\end{array}$ & $\begin{array}{l}55 \pm 5 \\
(36-84)\end{array}$ \\
\hline old & & & & & $\begin{array}{l}\text { Plasma insulin } \\
\text { (micro units } \\
\text { per ml) }\end{array}$ & $\begin{array}{l}24 \pm 2 \\
(15-32)\end{array}$ & $\begin{array}{c}81 \pm 12 \\
(18-210)\end{array}$ & $\begin{array}{c}75 \pm 10 \\
(28-129)\end{array}$ & $\begin{array}{c}49 \pm 9 \\
(26-88)\end{array}$ & $\begin{array}{c}31 \pm 8 \\
(19-47)\end{array}$ \\
\hline $\begin{array}{l}\text { Delayed } \\
\text { return }\end{array}$ & 7 & $\begin{array}{l}34 \\
(20-39)\end{array}$ & $2: 5$ & $\begin{array}{l}106 \\
(80-154)\end{array}$ & $\begin{array}{l}\text { Blood glucose } \\
(\mathrm{mg} \text { per } 100 \mathrm{ml})\end{array}$ & $\begin{array}{c}61 \pm 3 \\
(45-68) \\
\end{array}$ & $\begin{array}{l}108 \pm 7 \\
(92-130)\end{array}$ & $\begin{array}{l}102 \pm 9 \\
(68-136)\end{array}$ & $\begin{array}{l}87 \pm 6 \\
(66 \pm 105)\end{array}$ & $\begin{array}{l}79 \pm 4 \\
(62 \pm 95)\end{array}$ \\
\hline young & & & & & $\begin{array}{l}\text { Plasma insulin } \\
\text { (micro units } \\
\text { per ml) }\end{array}$ & $\begin{array}{c}21 \pm 3 \\
(15-35)\end{array}$ & $\begin{array}{c}51 \pm 4 \\
(34-61)\end{array}$ & $\begin{array}{c}47 \pm 4 \\
(22-56)\end{array}$ & $\begin{array}{c}41 \pm 4 \\
(28-56)\end{array}$ & $\begin{array}{c}37 \pm 3 \\
(20-46)\end{array}$ \\
\hline $\begin{array}{l}\text { Delayed } \\
\text { return }\end{array}$ & 8 & $\begin{array}{l}62 \\
(53-67)\end{array}$ & $3: 5$ & $\begin{array}{l}107 \\
(85-149)\end{array}$ & $\begin{array}{l}\text { Blood glucose } \\
\text { (mg per } 100 \mathrm{ml})\end{array}$ & $\begin{array}{c}64 \pm 3 \\
(51-73)\end{array}$ & $\begin{array}{l}109 \pm 8 \\
(79-150)\end{array}$ & $\begin{array}{l}124 \pm 6^{1} \\
(88-140)\end{array}$ & $\begin{array}{l}117 \pm 7 \\
(85=138)\end{array}$ & $\begin{array}{l}100 \pm 6^{2} \\
(80-140\end{array}$ \\
\hline old & & & & & $\begin{array}{l}\text { Plasma insulin } \\
\text { (micro units } \\
\text { per ml) }\end{array}$ & $\begin{array}{c}23 \pm 3 \\
(\mathbf{1 3}-\mathbf{3 6})\end{array}$ & $\begin{array}{c}46 \pm 6 \\
(22-79)\end{array}$ & $\begin{array}{c}52 \pm 3 \\
(38-63)\end{array}$ & $\begin{array}{c}66 \pm 9^{1} \\
(37-93)\end{array}$ & $\begin{array}{c}63 \pm 7^{2} \\
(35-84)\end{array}$ \\
\hline
\end{tabular}

$1 P<0.05$

$2 P<0.01$ in comparison with the concentration in the corresponding young subgroup.

non-obese and obese subgroups were compared (Table 2 ). In addition the mean fasting insulin concentration of the non-obese subgroup of Group II was significantly higher $(P<0.05)$ than that of the non-obese subgroup of Group I. The differences observed when the pooled results of Group I were compared with those of Group III were also observed when their respective non-obese and obese subgroups were compared. In ad-
Fig. 2), and in the old subgroup of Group III 90 $(P<0.05)$ and $120(P<0.01)$ min after glucose. The (insignificantly) higher insulin concentrations in the young subgroup of Group II are probably explained by the increased incidence of obesity in this subgroup.

Plasma-growth hormone. The mean plasma-growth hormone concentrations fasting and after glucose in Groups II and III did not differ significantly from 
those of Group I, apart from the fasting concentration of Group II, which was significantly lower $(P<0.05)$.

Since both the weight and sex of a subject may affect the plasma growth hormone concentration, the results were compared by dividing them into female or male, non-obese or obese, subgroups (Table 3). In nearly all the subgroups the fasting growth hormone concentration was lower in the obese than in the non obese subgroup from the same Group and sex, although, due to the small number and large scatter of obser-

Table 6. Plasma insulin concentration Blood glucose concentration

\begin{tabular}{|c|c|c|c|c|c|}
\hline \multirow[t]{2}{*}{ Group ${ }^{1}$} & \multicolumn{5}{|c|}{$\frac{\text { Plasma insulin concentration (microunits per } \mathrm{ml} \text { ) }}{\text { Blood glucose concentration (mg per } 100 \mathrm{ml} \text { ) }}$ and time after glucose administration } \\
\hline & min & 30 & $\begin{array}{l}60 \\
\min \end{array}$ & $\begin{array}{l}90 \\
\min \end{array}$ & $\begin{array}{l}120 \\
\min \end{array}$ \\
\hline $\begin{array}{l}\text { I Normal } \\
\text { non-obese }\end{array}$ & $\begin{array}{c}0.31 \pm 0.02 \\
(0.17 \pm 0.43)\end{array}$ & $\begin{array}{l}0.67 \pm 0.07^{4} \\
(0.22 \pm 1.1)\end{array}$ & $\begin{array}{l}0.63 \pm 0.08^{3} \\
(0.23-1.4)\end{array}$ & $\begin{array}{l}0.57 \pm 0.11^{2} \\
(0.19-1.9)\end{array}$ & $\begin{array}{l}0.45 \pm 0.05^{2} \\
(0.26=1.0)\end{array}$ \\
\hline $\begin{array}{l}\text { I Normal } \\
\text { obese }\end{array}$ & $\begin{array}{c}0.40 \pm 0.04 \\
(0.23-0.72)\end{array}$ & $\begin{array}{l}0.69 \pm 0.08^{3} \\
(0.30-1.3)\end{array}$ & $\begin{array}{l}0.73 \pm 0.10^{3} \\
(0.21-1.5)\end{array}$ & $\begin{array}{l}0.68 \pm 0.09^{3} \\
(0.39-1.4)\end{array}$ & $\begin{array}{l}0.61 \pm 0.07^{2} \\
(0.25=1.1)\end{array}$ \\
\hline $\begin{array}{l}\text { II Normal } \\
\text { glucosuric } \\
\text { non-obese }\end{array}$ & $\begin{array}{c}0.41 \pm 0.03 \\
(0.21-0.61)\end{array}$ & $\begin{array}{c}0.61 \pm 0.10 \\
(0.20-1.3)\end{array}$ & $\begin{array}{l}0.88 \pm 0.12^{3} \\
(0.28-1.5)\end{array}$ & $\begin{array}{l}0.73 \pm 0.09^{3} \\
(0.33-1.4)\end{array}$ & $\begin{array}{c}0.67 \pm 0.06^{3} \\
(0.24 \pm 0.95)\end{array}$ \\
\hline $\begin{array}{l}\text { II Normal } \\
\text { glucosuric obese }\end{array}$ & $\begin{array}{c}0.43 \pm 0.04 \\
(0.28-0.88)\end{array}$ & $\begin{array}{l}0.80 \pm 0.05^{4} \\
(0.53-1.4)\end{array}$ & $\begin{array}{r}0.84 \pm 0.08^{4} \\
(0.48-1.74)\end{array}$ & $\begin{array}{l}0.81 \pm 0.10^{3} \\
(0.45-1.8)\end{array}$ & $\begin{array}{r}0.57 \pm 0.08 \\
(0.18-1.4)\end{array}$ \\
\hline $\begin{array}{l}\text { III Delayed return } \\
\text { non-obese }\end{array}$ & $\begin{array}{c}0.29 \pm 0.02 \\
(0.16-0.54)\end{array}$ & $\begin{array}{c}0.39 \pm 0.02^{2} \\
(0.22-0.54)\end{array}$ & $\begin{array}{c}0.40 \pm 0.03^{2} \\
(0.16-0.66)\end{array}$ & $\begin{array}{r}0.47 \pm 0.05^{3} \\
(0.25-0.93)\end{array}$ & $\begin{array}{r}0.47 \pm 0.05^{3} \\
(0.17-0.94)\end{array}$ \\
\hline $\begin{array}{l}\text { III Delayed return } \\
\text { obese }\end{array}$ & $\begin{array}{c}0.46 \pm 0.06 \\
(0.27 \pm 1.1)\end{array}$ & $\begin{array}{c}0.57 \pm 0.08 \\
(0.28-1.6)\end{array}$ & $\begin{array}{c}0.59 \pm 0.08 \\
(0.28 \pm 1.4)\end{array}$ & $\begin{array}{c}0.58 \pm 0.06 \\
(0.29 \div 0.95)\end{array}$ & $\begin{array}{r}0.63 \pm 0.07 \\
(0.26 \pm 1.1)\end{array}$ \\
\hline
\end{tabular}

I Number of people per group and age, sex and weight composition of groups as in Table 2.

$2 P<0.05$.

$3 P<0.01$.

${ }_{4} P<0.001$ in comparison with fasting ratio in the same subgroup.

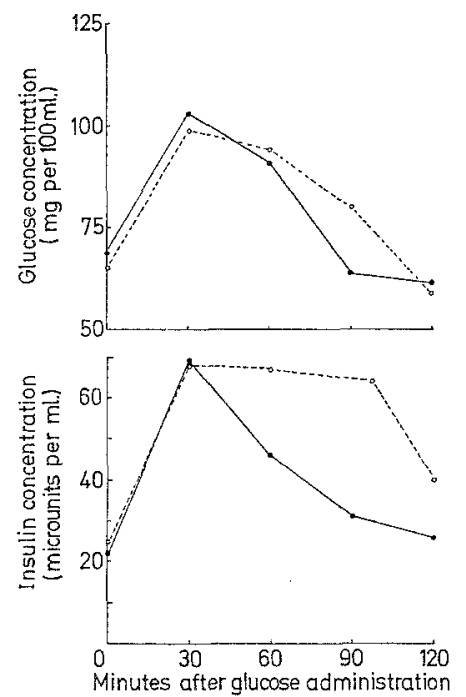

Fig. 2. Mean blood glucose and plasma insulin concentrations during $50 \mathrm{~g}$ oral glucose-tolerance tests in "young" (mean age 33) and "old" (mean age 54) people with normal glucose tolerance

Abscissa: lower graph; Minutes after glucose administration; 0, 30, 60, 90, 120.

Ordinate: upper graph; Glucose concentration (mg per $100 \mathrm{ml}) 50,75,100,125$.

lower graph; Insulin concentration (microunits per ml) $0,20,4060$.

$\longrightarrow$ young people

old people

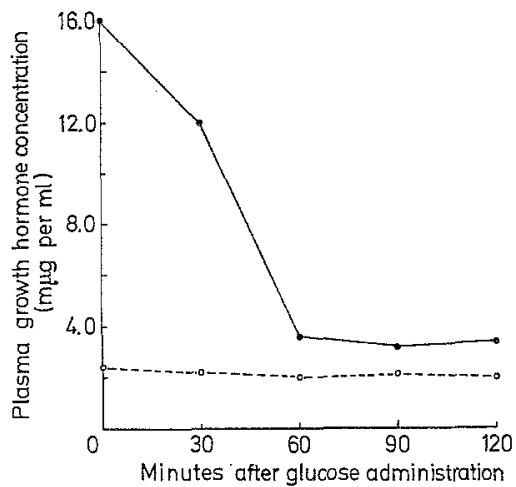

Fig. 3. Mean plasma growth hormone concentrations during $50 \mathrm{~g}$ oral glucose-tolerance tests in non-obese female and male people with normal glucose tolerance

Abscissa: Minutes after glucose administration, 0, 30, 60, 90,120 .

Ordinate: Plasma growth hormone concentration (mug per ml) $0,4.0,8.0,12.0,16.0$.

$\longrightarrow$ females

$\ldots$ males

vations, none of these differences were significant. In the subgroups of Group I the mean growth hormone concentrations of the female subjects were higher than those of the male, the differences being significant when the non-obese subjects were compared fasting $(P<0.01)$ and $30(P<0.01)$ and $60(P<0.05) \mathrm{min}$ after glucose (Fig. 3). On the contrary in the subgroups of Group III 
the mean growth hormone concentration of the nonobese female subjects were significantly lower than those of the non-obese males $60(P<0.05), 90(P<0.02)$ and $120(P<0.01)$ min after glucose.

The mean plasma growth hormone concentrations also varied according to whether subjects belonged to Groups I, II or III. The mean concentrations observed in Group II (all male) were significantly: a) lower fasting when the non-obese were compared with the non obese males of Group III $(P<0.05)$; b) $30(P<0.01)$, $60(P<0.05)$ and $90(P<0.01)$ min after glucose when the obese were compared with the obese males of Group III; c) fasting $(P<0.05)$ and $30(P<0.05)$ and $90(P<0.05)$ min after glucose when the obese were compared with the obese males of Group I. The mean concentrations observed in the non-obese male subgroup of Group III were significantly higher than in the non obese male subgroup of Group I fasting $(P<0.01)$, and $30(P<0.05) 60(P<0.02), 90(P<0.01)$ and $120(P<0.01) \mathrm{min}$ after glucose (Fig. 4).

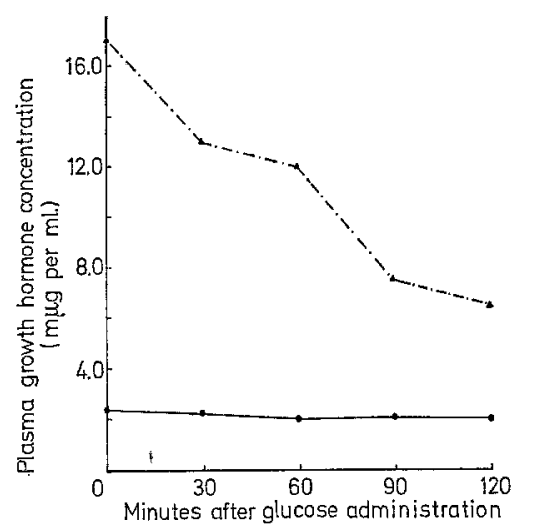

Fig. 4 - Mean plasma growth hormone concentrations during $50 \mathrm{~g}$ oral glucose-tolerance tests in non-obese males with normal glucose tolerance (Group I) and with a minor abnormality of glucose tolerance (Group III)

Abscissa: Minutes after glucose administration, $0,30,60$, $90,120$.

Ordinate: Plasma growth hormone concentrations (mug per ml) $0,4.0,8.0,12.0,16.0$.

$\longrightarrow$ males with normal glucose tolerance (Group I) males with a minor abnormality of glucose tolerance (Group III)

Plasma-insutin/blood-glucose. Insulin: glucose ratios were calculated, for the individuals whose results are summarised in Table 2 , in the fasting state and at each interval after glucose. The means ( \pm standard error and range) are given in Table 4 . There was a significant rise in the ratio after glucose in each of the subgroups except in the obese subgroup of Group III. There was a tendency for the insulin: glucose ratio to be higher in the obese subjects, but this difference was only significant when the obese were compared with the non obese subgroup of Group III fasting and 30 and $60 \mathrm{~min}$ after glucose $(P<0.05)$. In comparison with the non obese subgroup of Group I: a) the non-obese subgroup of Group II showed a higher mean insulin: glucose ratio fasting and $120 \mathrm{~min}$ after glucose $(P<0.05)$; b) the non obese subgroup of Group III showed a lower mean ratio $30(P<0.01)$ and $60(P<0.05)$ min after glucose (Fig. 5).

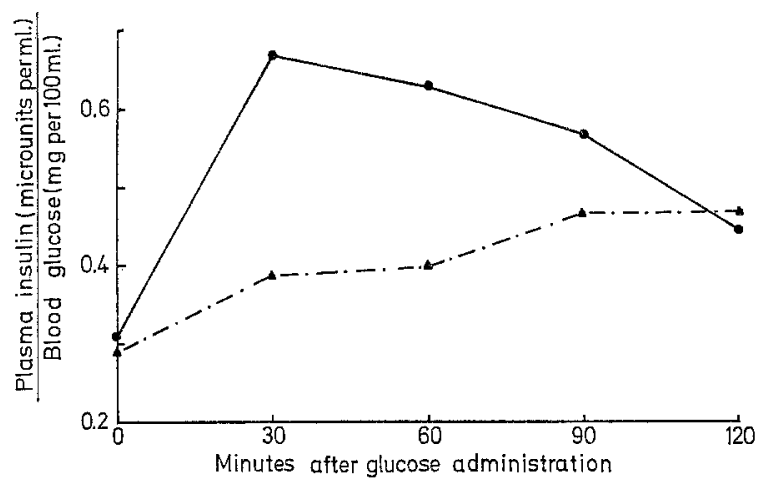

Fig. 5. Mean insulin glucose ratios during $50 \mathrm{~g}$ oral glucosetolerance tests in non-obese people with normal glucose tolerance (Group I) and with a minor abnormality of glucose (Group III)

Abscissa: Minutes after glucose administration, 0, 30, 60, $90,120$.

Ordinate: Plasma insulin (microunits per $\mathrm{ml}$ ) $0.2 ; 0.4 ; 0.6$. Blood glucose (mg per $100 \mathrm{ml}$ )
$\longrightarrow$ normal people (Group I)
$\stackrel{\longrightarrow}{\longrightarrow}$ people with minor abnormalities of glucose tolerance

\section{Discussion}

Effects of weight, sex and age. The effects of weight, sex and age in Group I together with the relatively small differences of mean weight involved confirm and extend the findings of others (for review see HaLis, 1967 a).

Recently Chuouverakis et al. (1967) have reported that in a group of individuals with normal glucose tolerance, the serum insulin concentration increased. with increasing age in the absence of a notable change in glucose tolerance. However, the subjects studied also showed an increase in weight with increasing age. Serum insulin and blood sugar concentrations were measured fasting and at 1 and $2 \mathrm{~h}$ after glucose. In a borderline diabetic group they found no effect of age on serum insulin concentration. Our results in individuals with normal glucose tolerance show an effect of age on glucose tolerance which is independent of weight and clearly seen 90 min after glucose (Table 5; Fig. 2). A similar pattern was observed in subjects with a minor abnormality of glucose tolerance. These results show, therefore, that older subjects when compared with younger subjects of the same weight, with the same fasting and $30 \mathrm{~min}$ blood sugar and plasma insulin concentration, show a worse glucose tolerance, and this appears to be due to insensitivity to endogenous insulin. This affect of age is seen whether the glucose tolerance is normal or slightly abnormal. 
These findings emphasize the need for very careful matching of groups for weight, sex and age, in searching for an effect of additional factors on the parameters studied.

Subjects with normal glucose tolerance and glucosuria (Group II). The importance of these subjects lies in the fact that they are detected in large numbers by diabetic surveys (MTTCHeLl and StradSs, 1964). If it is true that many persons so detected are destined to become diabetics, it is clearly important that they should be investigated, because they may throw light on metabolic abnormalities present before the development of overt diabetes. The type of glucose tolerance found in these subjects (characterized by an exaggerated early rise in glucose and insulin, a tendency to late hypoglycaemia and a normal insulin : glucose ratio) resembles that seen in alimentary functional hypoglycaemia (CONN and SELTzER, 1955) and after gastrectomy (Roth and Mrade, 1965). The way the alimentary tract deals with the glucose load may not be the only explanation of the differences observed, since the fasting plasma insulin concentration of the non-obese subgroup was higher, and the fasting plasma growth hormone of the obese subgroup was lower, than that of the corresponding subgroup of normal subjects without glucosuria.

Subjects with 'delayed return' (Group III). Due to the way in which subjects in this category were selected, the glucose tolerances, of the non-obese and obese subgroups of Group III were very similar (Table 2). It is well known that obesity is a factor causing a deterioration of glucose tolerance and diminished sensitivity to endogenous insulin (for a recent discussion see PERLEY and KIPNIS, 1966). Therefore it is likely that the major factor causing reduced glucose tolerance in the obese subgroup of Group III is obesity; and it is not surprising that the results obtained in this subgroup differed considerably from those of the non-obese subgroup. The latter are of greater interest since they may indicate factors other than obesity which are of importance in the production of minor abnormalities of glucose tolerance. The most obvious of these is the delay in the rise of insulin in response to oral glucose, and shown most clearly by the fact that the insulin: glucose ratio (or "insulinogenic index" as it has been termed by SeLtzer and Harris, 1964) $30 \mathrm{~min}$ after glucose was almost half that seen in the normal non obese subjects (Fig. 5). The response to endogenous insulin may be assessed by a comparison of the area under the curve of insulin concentration with that of normal subjects (YALOW and BERSON, 1960; PERLEY and KIPNIS, 1966). The area under the curve (Fig. 1) of the non-obese subjects with delayed return was $29 \%$ higher than that of the corresponding normal subjects. However, a better comparison of the sensitivity of these two subgroups to endogenous insulin would be given by the comparison of the areas under the respective insulin curves up to the time at which the blood sugar concentration is restored to the fasting level. In the non-obese subjects with delayed return, this would appear by extrapolation to be twice that of the normal subjects. There is therefore also evidence of insulin resistence in these individuals.

Seltzer et al. (1967) studied insulin secretion in response to an oral glucose load of $1.75 \mathrm{~g}$ of glucose per $\mathrm{kg}$ of "desirable" body weight in a group of mild dia.betics. In their study the mean age of the normal subjects was less than that of the diabetics (33 and 47 years respectively) and the abnormality of glucose tolerance of the mild diabetics was greater than in the present study. Nevertheless, the results of the two studies are in good agreement. SELTzER et al. found a delay in the rise of plasma insulin concentration after oral glucose but a higher absolute insulin output in normal weight subjects with mild diabetes.

In the non-obese male subjects of this group there appeared to be an abnormality of plasma growth hormone concentration, although the small number of subjects studied, together with the lability of plasma growth hormone concentration in response to exercise (RoTH et al., 1963) and stress (GREENWOOD and LANDON, 1966) means that further studies of this aspect are required.

The Aetiology of Diabetes Mellitus. These results are only of relevance to the aetiology of diabetes in so far as they represent observations in individuals who are destined to develop clinical diabetes. $J_{A C K s o N}$ and Woolf (1957) and FaJANs and Conn (1959) have emphasised the value of minor abnormalities of glucose tolerance in predicting the later onset of diabetes, but the degree of abnormality involved was rather greater than that of Group III in this study. Recently Ricketrs et al. (1966) have observed in "prediabetics" (defined mainly by a strong genetic predisposition to diabetes) a glucose tolerance very similar to that of the present Group III. In addition, out of a small group of subjects with this type of glucose tolerance studied previously (HaLES and RANDLE, 1963a), one has now developed diabetes with ketosis (HALES, 1967b). If the non-obese subjects in Group III are destined to become diabeties, then the implications of the present study are that there is, very early in the development of the disease, an abnormality both of insulin secretion in response to glucose and sensitivity to endogenous insulin. The scatter of the results obtained suggests that the contribution of the two factors varies with the individual. Insulin deficiency alone is unlikely to be the cause of these $a b$ normalities since individuals with severe insulin deficiency due to chronic pancreatitis have been observed to have only minor abnormalities of glucose tolerance (PETERs et al., 1966).

If individuals with a normal glucose tolerance and glucosuria become diabetic as has been suggested (ACKERMAN et al., 1958), then there is no evidence of 
an abnormality of insulin secretion at this stage of the disease. The clear differences between these subjects (Group II) and Group III, and the possibility of an abnormality of growth hormone secretion in some subjects, indicate the need for further investigation of larger numbers of these individuals such that subjects can be matched for weight, sex and age, and multiple observations carried out simultaneously. Only in this way can the influence of the many factors now known to be important in the determination of glucose tolerance be evaluated for their role in the production of clinical diabetes.

Acknowledgements. We are grateful to The British Diabetic Association for a research grant (C.N.H., F.L.M., and W.T.S.), to Burroughs Wellcome Research Laboratories, Beckenham for the gift of ox insulin and rabbit anti-(guinea pig serum) antiserum, to Mr. J.C.G. PEarsoN and the Department of Public Health, University of St. Andrews and to Mr. D.E. Bowrer for assistance in the preparation of the data for statistical analysis by computer.

\section{References}

Ackeryan, I.P., S.S. Fajans, and J.W. Conn: The development of diabetes mellitus in patients with nondiabetic glycosuria. Clin. Res. Proc. 6, 251 (1958).

Chlovverakis, C., R.J. Jarreit, and H. KEEN : Glucose tolerance, age and circulating insulin. Lancet $1967 \mathrm{I}$, $806-809$.

ConN, J.W., and H.S. SELtzer: Spontaneous hypoglycaemia. Amer. J. Med. 19, 460-478 (1955).

Discombe, G.: An inexpensive method for the determination of true glucose in blood and other fluids by the Auto-Analyzer. J. clin. Path. 16, 170-172 (1959).

FAJANS, S.S., and J.W. CoNN: The early recognition of diabetes mellitus. Ann. N.Y. Acad. Sci. 82, 208-218 (1959).

Gremewood, F.C., and J. Landon: Assessment of hypothalamic pituitary function in endocrine disease. J. clin. Path. 19, 284-292 (1966).

HaLes, C.N., and P.J. Randex: Effects of low-carbohydrate diet and diabetes mellitus on plasma concentrations of glucose, non-esterified fatty acid, and insulin during oral glucose-tolerance tests. Lancet 1963 a I, 790.

- - Immunoassay of insulin with insulin-antibody preeipitate. Biochem. J. 88, 137-146 (1963b).

- Hormonal disturbances in diabetes, in Carbohydrate Metabolism and its Disorders. Ed. F. Dickens, P.J.
Randle, and W.J. Whelan. London: Academic Press, in press $(1967 \mathrm{a})$.

- Plasma glucose non-esterified fatty acid, glycerol and insulin concentrations four years before the onset of diabetic ketosis. Lancet 1967 II, 389-390.

HUNTER, W.M., and F.C. GREENwOOD: A radio-immunoelectrophoretic assay for buman growth hormone. Biochem. J. 91, 43-56 (1964).

JACKSON, W.P.U., and N. WoolF: Further studies in prediabetes. Lancet 1957 I, 614-617.

Kemsuey, W.F.F.: Body weight at different ages and heights. Ann. Eugen. 16, 316-334.

McDonatd, G.W., G.F. Fisher, and C. Burnham: Reproducibility of the oral glucose tolerance test. Diabetes 14, 473-480 (1965).

Mitoheli, F.L., and W.T. Strauss: Relation of postprandial blood-glucose level to the oral glucose tolerance curve. Lancet 1964 I, 1185-1189.

Perley, M., and D.M. KIPNIS: Plasma insulin responses to glucose and tolbutamide of normal weight and obese diabetic and non diabetic subjects. Diabetes 15, 867874 (1966).

Peters, N., A.P. Diok, C.N. Hales, D.H. OrReil, and M. SARNER: Exocrine and endocrine pancreatic function in diabetes mellitus and chronic pancreatitis. Gut. 7, 277-281 (1966).

Rickets, H.T., R.A. CherRy, and L. Kirstenns: Biochemical studies of "prediabetes". Diabetes 15, $880-$ 888 (1966).

Roth, D.A., and R.C. Mrade: Hyperinsulinism-hypoglycaemia in the post gastrectomy patient. Diabetes 14, 526-530 (1965).

ROTH, J., S.M. GIICK, R.S. YAIOW, and S.A. BERSON: Secretion of human growth hormone: Physiologic and experimental modification. Metabolism 12, 577-579 (1963).

Seltzer, H.S., E.W. Allen, A. L. Herron, Jr., and M.T. BRENNAN: Insulin secretion in response to glycemic stimulus: relation of delayed initial release to carbohydrate intolerance in mild diabetes mellitus. J. clin. Invest. 46, 323-335 (1967).

-, and V.L. HARRIS: Exhaustion of insulogenic reserve in maturity-onset diabetic patients during prolonged and continuous hyperglycaemic stress. Diabetes 13,6-13 (1964).

YALOW, R.S., and S.A. Berson: Immunoassay of endogenous plasma insulin in man. J. clin. Invest. 39, $1157-1175$ (1960).

C.N. Hales, M.A., M.B., Ph.D. Cantab. Lecturer

Department of Biochemistry

University of Cambridge

Cambridge, England 